\title{
Proper Fuzzification of Prime Ideals of a Hemiring
}

\author{
H. V. Kumbhojkar \\ 403 Poorvarang Apartments, 13th Lane, Kolhapur 416008, Maharashtra, India \\ Correspondence should be addressed to H. V. Kumbhojkar, hvk_maths@yahoo.co.in
}

Received 26 April 2012; Accepted 8 October 2012

Academic Editor: Sendren Sheng-Dong Xu

Copyright ( 2012 H. V. Kumbhojkar. This is an open access article distributed under the Creative Commons Attribution License, which permits unrestricted use, distribution, and reproduction in any medium, provided the original work is properly cited.

Prime fuzzy ideals, prime fuzzy $k$-ideals, and prime fuzzy $h$-ideals are roped in one condition. It is shown that this way better fuzzification is achieved. Other major results of the paper are: every fuzzy ideal (resp., $k$-ideal, $h$-ideal) is contained in a prime fuzzy ideal (resp., $k$-ideal, $h$-ideal). Prime radicals and nil radicals of a fuzzy ideal are defined; their relationship is established. The nil radical of a fuzzy $k$-ideal (resp., an $h$-ideal) is proved to be a fuzzy $k$-ideal (resp., $h$-ideal). The correspondence theorems for different types of fuzzy ideals of hemirings are established. The concept of primary fuzzy ideal is introduced. Minimum imperative for proper fuzzification is suggested and it is shown that the fuzzifications introduced in this paper are proper fuzzifications.

\section{Introduction}

This paper is, in some sense, an extended version of the article "On Fuzzification of Prime Ideals with Special Reference to Semirings" in SciTopics and something more.

Several attempts have been made to fuzzify the concepts of prime ideals $/ k$-ideals $/ h$-ideals of a semiring [1-7], prime ideals of a ring [8-15], and prime ideals of a semigroup [16-18]. We have discussed elsewhere [6], in detail, the deficiencies in the definition of a prime fuzzy $h$-ideal proposed in [7]. The definition suffers from three major drawbacks. First, it is very restrictive in the sense that the fuzzy $h$-ideals, which are prime according to the definition, are 2-valued function. Secondly, since one of the two values is always 1 (the greatest element of the lattice), the function is determined by only one value, thus, severely curtailing its fuzziness. Third, when the zero element of the valuation lattice is not a prime element (and this happens in many important lattices), even the characteristic function of a prime ideal fails to be a prime fuzzy ideal. The technique adopted for the fuzzification by Zhan and Dudek in [7] and by others in $[1-3,5]$ is identical. Therefore, their prime fuzzy ideals inherit the same drawbacks. In [6] we have redefined prime fuzzy left $h$-ideal so that these deficiencies are completely removed. (It should be thankfully mentioned that one of the referees of the present paper has pointed out that in [4] two similar definitions of prime fuzzy ideal are stated. However, while proving major results of the paper, only 2-valued prime fuzzy ideals are used.) In this paper, we show that the problem of fuzzification of left ideal, left $k$-ideal, and left $h$-ideal need not be tackled separately. One single condition governs all the three. We also "refine" our definitions so that they look more compact, elegant, and easy for application. We prove that every proper fuzzy ideal (resp., $k$-ideal, $h$-ideal) is contained in a prime fuzzy ideal (resp., $k$-ideal, $h$-ideal). We introduce the concepts of fuzzy prime radical (or to be more precise, prime radical of a fuzzy ideal) and fuzzy nil radical (or nil radical of a fuzzy ideal), and fuzzy primary ideal. The prime and the nil radicals of a fuzzy $k$-ideal coincide when the valuation lattice is linearly ordered (e.g., when it is $[0,1]$ ). An analogous result holds for fuzzy $h$-ideals. We establish a correspondence between fuzzy ideals (resp., $k$-ideals, $h$-ideals) of a hemiring and those of its homomorphic image. The correspondence preserves prime, semiprime, and primary fuzzy ideals $/ k$ ideals $/ h$-ideals. Fuzzifications introduced in this paper can be labeled as "proper fuzzifications".

\section{Preliminaries}

2.1. Ideals of a Semiring. In the following discussion, $(S,+, \cdot)$ stands for a semiring. That is, $(S,+)$ is a commutative monoid having identity element 0 and $(S, \cdot)$ is a semigroup satisfying the following identities: $a(b+c)=a b+a c,(a+$ $b) c=a c+b c$, and $0 \cdot x=0=x \cdot 0$. A commutative semiring with unity is a semiring $(S,+, \cdot)$ such that $(S, \cdot)$ is 
a commutative monoid. We denote the identity element of $(S, \cdot)$ by 1 . With abuse of notation, we denote $(S,+, \cdot)$ by $S$. A left ideal $A$ of $S$ is a nonempty set $A$ which is closed under the addition of $S$ and is such that, for all $x \in S$ and $a \in A$ we have $x a \in A$. A left ideal $A$ of $S$ is called a left $k$-ideal, if for all $x \in S, x+a \in A$, and $a \in A \Rightarrow x \in A$. It is called a left $h$-ideal, if for all $x, z \in S, x+a+z=b+z$, and $a, b \in A \Rightarrow x \in A$. A right ideal (resp., $k$-ideal, $h$-ideal) is similarly defined. Whenever a statement is made about left ideals, it is to be understood that the analogous statement is made about right ideals. An ideal is one, which is both right and left ideal. A left ideal $P$ is called prime left ideal, if it satisfies the following conditions:

(i) $P \neq S$ and

(ii) for all left ideals $A \& B$ of $S$, we have

$$
A B \subseteq P \Longrightarrow \text { either } A \subseteq P \text { or } B \subseteq P .
$$

It is natural to call $P$ a $k$-prime (resp., $h$-prime) left ideal, if the condition (I) holds for left $k$-ideals (resp., $h$-ideals) $A$ and $B$.

Clearly, every prime left ideal is $k$-prime and every $k$ prime left ideal is $h$-prime. However, as will be seen in Example 1, the reverse implications, in general, are not true.

Example 1. (a) If $S=\{0, \alpha, \beta, 1\}$ is the Boolean lattice of four elements, then 0 is not a $k$-prime ideal, as the condition (I) fails for $k$-ideals $A=\{0, \alpha\}$ and $B=\{0, \beta\}$. However, $S$ being the only $h$-ideal of $S, 0$ is $h$-prime. Clearly, 0 is neither prime nor an $h$-ideal.

(b) Consider the semiring $S=\{0,1,2,3\}$, where the binary operations $\oplus$ and $\otimes$ are defined as follows: $a \oplus b=$ $\operatorname{Min}\{a+b, 3\}$ and $a \otimes b=\operatorname{Min}\{a b, 3\}$. One can easily see that $S$ has only three proper ideals, namely, $0, A=\{0,2,3\}$, and $B=\{0,3\}$. Since we have $A A \subseteq B$ and $A \nsubseteq B, B$ is not a prime ideal. However, 0 and $S$ being the only $k$-ideals of $S$, one can see that $B$ is a $k$-prime ideal. Again, $B$ is neither prime nor a $k$-ideal.

We shall soon see that the concepts of primeness and $k$ primeness (resp., $h$-primeness) coincide for $k$-ideals (resp., $h$-ideals).

Proposition 2 (see $[5,7]$ ). If $S$ is a semiring and $A$ and $B$ are left ideals of $S$, then $k(A B)=k(k(A) k(B))$ and $h(A B)=$ $h(h(A) h(B))$, where $k(A)$ and $h(A)$, respectively, denote $k$ closure and h-closure of $A$.

Using Proposition 2 we get the following.

Theorem 3. Let $P$ be a proper left $k$-ideal (resp., $h$-ideal) of a semiring $S$. The following statements are equivalent.

(a) $P$ is prime.

(b) $P$ is k-prime (resp., h-prime).

(c) For all $a, b \in S, a S b \subseteq P$ implies $a \in P$ or $b \in P$.

Proof. We prove the statement: “(b) implies (c)”, for $h$-ideals. Suppose $P$ is a proper $h$-prime left $h$-ideal such that $a S b \subseteq P$ for $a, b \in S$. Clearly, we have $S a S b \subseteq P$. Our first claim is that for $A=h(S a)$ and $B=h(S b)$, where $h(I)$ stands for the $h$-closure of a left ideal $I$ of $S$, we have $A B \subseteq P$. Suppose we have $x \in A$ and $y \in B$. Then for some $s, t, u, v, z, z^{\prime}$, in $S$, we have $x+s a+z=t a+z$ and $y+u b+z^{\prime}=v b+z^{\prime}$ and, therefore, the equalities: $a y+a u b+a z^{\prime}=a v b+a z^{\prime}$, and $x y+s a y+z y=t a y+z y$. As $a u b$ and $a v b$ are elements of $P$ and $P$ is an $h$-ideal, $a y$ is in $P$. Therefore, say, tay and consequently, $x y$ are in $P$. It, then, follows that $A B \subseteq P$ and, $P$ being $h$-prime, we have either $A \subseteq P$ or $B \subseteq P$. Suppose $A \subseteq P$. If $\langle a\rangle$ is the left ideal generated by $a$, then we have $\langle a\rangle\langle a\rangle \subseteq S a$ and hence, $h(\langle a\rangle) h(\langle a\rangle) \subseteq h(h(\langle a\rangle) h(\langle a\rangle))=h(\langle a\rangle\langle a\rangle) \subseteq$ $h(\mathrm{~S} a)=A \subseteq P$. Since $P$ is $h$-prime, we have $h(\langle a\rangle) \subseteq P$ and consequently, $a \in P$.

Using Zorn's Lemma one can prove the following.

Theorem 4. Every proper ideal (resp., $k$-ideal, $h$-ideal) of a commutative hemiring $S$ with unity is contained in a prime ideal (resp., $k$-ideal, $h$-ideal) of $S$.

Theorem 5. If $T$ is a multiplicatively closed set in a commutative hemiring $S$ with unity, disjoint from an ideal (resp., $k$-ideal, $h$-ideal) I of $S$, then there exists a prime ideal (resp., $k$-ideal, $h$ ideal) $P$ of $S$ such that $I \subseteq P$ and $P \cap T=\varnothing$.

2.2. Prime Ideals of $\mathbf{N}$. In the hemiring $\mathbf{N}$ of nonnegative integers, obviously, an ideal $\mathbf{I}$ is a $k$-ideal if and only if it is an $h$-ideal. Moreover, $\mathbf{I}$ is a $k$-ideal if and only if $\mathbf{I}=n \mathbf{N}$ for some $n \in \mathbf{N}$. The prime $k$-ideals of $\mathbf{N}$ are either $p \mathbf{N}$ where $p$ is a prime number in $\mathbf{N}$ or the zero ideal. For each prime $p$ the ideal $p \mathbf{N}$ is a maximal $k$-ideal [19]. Clearly, $p \mathbf{N}$ is not a maximal ideal of $\mathbf{N}$.

Proposition 6. Let $p$ be a prime integer in $\mathbf{N}$ and $\mathbf{P}=\mathbf{N} \sim$ $\{1\}$. There is no prime ideal $\mathbf{I}$ of $\mathbf{N}$ such that $p \mathbf{N} \subset \mathbf{I} \subset \mathbf{P}$.

Proof. We first prove that the proposition holds for $p=2$. Assuming the contrary, let I be a prime ideal such that $2 \mathrm{~N} \subset$ $\mathbf{I} \subset \mathbf{P}$. Let $x$ be the smallest element of $\mathbf{I} \sim 2 \mathbf{N}$. Then $x=2 n+$ 1 for some positive integer $n$. Let $\mathbf{J}=\{z \in \mathbf{N} \mid z \geq 2 n\} \cup\{0\}$. Since $2,2 n$ and $2 n+1$ are in $\mathbf{I}$ and $\mathbf{I}$ is closed under addition, we have $\mathbf{J} \subset \mathbf{I}$. Clearly, if $n=1$, then $\mathbf{J}=\mathbf{P}$. Therefore, we have $n \neq 1$ and $x \geq 5$. Consider $y=3$. For sufficiently large value of $s$, we have $y^{s}$ is in $\mathbf{J}$ and hence, in $\mathbf{I}$. Since $\mathbf{I}$ is a prime ideal, we have $y \in \mathbf{I}$. This contradicts the assumption that $x$ is the smallest element of $\mathbf{I} \sim 2 \mathbf{N}$. Therefore, $\mathbf{I}$ is not a prime ideal.

Consider a prime integer $p \geq 3$ and a prime ideal I such that $p \mathbf{N} \subset \mathbf{I} \subset \mathbf{P}$. Let $x$ be the smallest element of $\mathbf{I} \sim p \mathbf{N}$. Then, $x=p n+r$ for some $n \in \mathbf{N}$ and $r=1,2, \ldots, p-1$. Consider $x=p n+1$. Clearly, $n \neq 0$ and thus, we have $x \geq 4$. Let $\mathbf{J}=\{z \in \mathbf{N} \mid z \geq(p-1) p n\} \cup\{0\}$. Observe that for all $r=1,2, \ldots, p-1$ we have $(p-1) p n+r=(p-(r+1)) p n+$ $r(p n+1)$. Therefore $(p-1) p n+r \in I$. However, I contains $p \mathbf{N}$ and therefore, J. Now set $y=2$. Since we assume $\mathbf{I}$ to be a prime ideal, we get $y \in \mathbf{I} \sim p \mathbf{N}$. This contradicts the choice of $x$ as the smallest element in $\mathbf{I} \sim p \mathbf{N}$. Therefore, $x \neq p n+1$. Consider $x=p n+r$ for $1<r \leq p-1$. Then, clearly, we have 
$x \geq 2$. We claim that $x \neq 2$. If $x=2$, then $2 \mathrm{~N} \subseteq \mathbf{I}$. Obviously, we have $2 \mathbf{N} \not \subset \mathbf{I}$. On the other hand, if $2 \mathbf{N}=\mathbf{I}$, then, we get the absurd result that $p \mathbf{N} \subseteq 2 \mathbf{N}$ for $p \neq 2$. Now set, as before, $y=2$ to get the contradiction to the assumption that $x$ is the smallest element of $\mathbf{I} \sim p \mathbf{N}$ and complete the proof.

Theorem 7. $\mathbf{P}=\mathbf{N} \sim\{1\}$ is the only prime ideal of $\mathbf{N}$ which is not a $k$-ideal (resp., an h-ideal).

Proof. One easily observes that $\mathbf{P}$ is a prime ideal and is not a $k$-deal. Let $\mathbf{I}$ be any other ideal of $\mathbf{N}$, which is not a $k$ ideal. Clearly, then, we have $0 \subset \mathbf{I} \subset \mathbf{P}$. Therefore, there exist $x \in \mathbf{I}$ such that $0 \neq x \neq 1$. Let $x=p_{1}^{\alpha 1}, \ldots, p_{n}^{\alpha n}$ be the prime factorization of $x$. If $\mathbf{I}$ is prime, there is at least one prime integer $p$ in $\mathbf{I}$. Therefore, we have $p \mathbf{N} \subseteq \mathbf{I} \subset \mathbf{P}$. As $\mathbf{I}$ is not a $k$ ideal we have $p \mathbf{N} \neq \mathbf{I}$. On the other hand, by Proposition 6 we cannot have a prime ideal I such that $p \mathbf{N} \subset \mathbf{I} \subset \mathbf{P}$. Therefore, I is not a prime ideal.

2.3. Fuzzy Ideals of a Semiring. Throughout this paper $L$ stands for a complete Heyting algebra, that is, a complete lattice such that for all subsets $T$ of $L$ and all $b \in L, \vee\{a \wedge b \mid$ $a \in T\}=(\vee\{a \mid a \in T\}) \wedge b$ and $\wedge\{a \vee b \mid a \in T\}=$ $(\wedge\{a \mid a \in T\}) \vee b$. An $L$-fuzzy subset (or simply an $L$ fuzzy set) $A$ of a set $X$ is a function $A: X \rightarrow L$; a fuzzy set is an $L$-fuzzy set when $L$ is the unit interval $[0,1]$. If $\alpha \in L$, then the set $\{x \in X \mid A(x) \geq \alpha\}$ is called $\alpha$-level cut or in short $\alpha$-cut of $A$ and is denoted by $A_{\alpha}$. The strict $\alpha$-level cut of $A$ is the set $A_{\alpha+}=\{x \in X \mid A(x)>\alpha\}$. An $L$-fuzzy left ideal $J$ of $S$ is an $L$-fuzzy set $J: S \rightarrow L$ such that for all $a, b \in S$ the following conditions are satisfied: (i) $J(a+b) \geq J(a) \wedge J(b)$, (ii) $J(a b) \geq J(b)$. An $L$-fuzzy left ideal $J$ of $S$ is called an $L$-fuzzy left $k$-ideal, if the following condition is satisfied: $x+a=b \Rightarrow J(x) \geq J(a) \wedge J(b)$ for all $x, a, b, \in S$. It is an $L$-fuzzy left $h$-ideal, if $x+a+z=b+z \Rightarrow J(x) \geq$ $J(a) \wedge J(b)$ for all $x, a, b, z \in S$. An $L$-fuzzy right ideal (resp., $k$-ideal, $h$-ideal) is similarly defined. Whenever a statement is made about $L$-fuzzy left ideals, it is to be understood that the analogous statement is made about an $L$-fuzzy right ideals. An $L$-fuzzy ideal is one, which is both $L$-fuzzy right and $L$ fuzzy left ideal.

\section{Prime Fuzzy Ideals}

We defined $L$-fuzzy prime $h$-ideal in [6]. We extend the definition to $L$-fuzzy ideals and $k$-ideals.

Definition 8. An $L$-fuzzy left ideal (resp., $k$-ideal, $h$-ideal) $P$ of $S$ is called a prime $L$-fuzzy left ideal (resp., $k$-ideal, $h$-ideal), if it is nonconstant and, for all $a, b \in S$ and $\alpha \in L$, the following condition is satisfied:

$$
P(a s b) \geq \alpha, \quad \forall s \in S \Longrightarrow P(a) \geq \alpha \text { or } P(b) \geq \alpha .
$$

Proposition 9. A nonconstant L-fuzzy left ideal (resp., $k$-ideal, $h$-ideal) $P$ of $S$ is prime if and only if its every nonempty level cut of $P$ is either a prime left ideal (resp., $k$-ideal, $h$-ideal) of $S$ or $S$ itself.
Corollary 10. A left ideal (resp., k-ideal, $h$-ideal) $\mathbf{P}$ of $S$ is prime if and only if its characteristic function $\chi_{P}$ is an $L$ fuzzy prime left ideal (resp., $k$-ideal, $h$-ideal) for every complete Heyting algebra L.

Proposition 9 is proved for $L$-fuzzy left h-ideal in [6].

Let $P$ be an $L$-fuzzy prime (two sided) ideal of $S$. Then $P(a s b) \geq P(a) \vee P(b)$ for all $s \in S$ and, therefore, $\wedge\{P(a s b) \mid$ $s \in S\} \geq P(a) \vee P(b)$. On the other hand, for $\wedge\{P(a s b) \mid s \in$ $S\}=\alpha$, we have:

$$
\begin{aligned}
& P(a s b) \geq \alpha, \quad \forall s \in S \Longrightarrow P(a) \geq \alpha \text { or } P(b) \geq \alpha \\
& \Longrightarrow P(a) \vee P(b) \geq \alpha=\wedge\{P(a s b) \mid s \in S\} .
\end{aligned}
$$

Therefore, $\wedge\{P(a s b) \mid s \in S\}=P(a) \vee P(b)$.

Let, further, $P(a) \geq \alpha$. Then, $P(a) \geq \alpha=\wedge\{P(a s b) \mid s \in$ $S\} \geq\{P(a) \vee P(b)\} \geq P(b)$.

Thus, $P(S)$ is totally ordered.

Conversely, let $P(S)$ be totally ordered and $\wedge\{P(a s b)$ | $s \in S\}=P(a) \vee P(b)$. Then,

$$
\begin{gathered}
P(a s b) \geq \alpha, \quad \forall s \in S \Longrightarrow P(a) \vee P(b)=\wedge\{P(a s b) \mid s \in S\} \\
\geq \alpha \Longrightarrow P(a) \geq \alpha \text { or } P(b) \geq \alpha .
\end{gathered}
$$

Therefore, $P$ is a prime $L$-fuzzy ideal.

This leads to the following elegant characterizations of prime fuzzy ideals.

Proposition 11. Let $P$ be a nonconstant L-fuzzy ideal (resp., $k$-ideal, $h$-ideal) of $S$, and $a, b \in S$.

(1) $P$ is prime if and only if $\wedge\{P(a s b) \mid s \in S\}=P(a) \vee$ $P(b)$ and $P(S)$ is totally ordered.

(2) Let $S$ be commutative hemiring with unity. $P$ is prime if and only if $P(a b)=P(a) \vee P(b)$ and $P(S)$ is totally ordered.

(3) A nonconstant fuzzy ideal (resp., $k$-ideal, $h$ - ideal) $P$ is prime if and only if $\operatorname{Inf}\{P(a s b) \mid s \in S\}=$ $\operatorname{Max}\{P(a), P(b)\}$.

(4) Let $S$ be commutative hemiring with unity. A nonconstant fuzzy ideal (resp., $k$-ideal, $h$-ideal) $P$ is prime if and only if $P(a b)=\operatorname{Max}\{P(a), P(b)\}$.

The following example shows that the condition that $P(S)$ is totally ordered is necessary for $P$ to be prime.

Example 12. Let $L=\{0, \alpha, \beta, 1\}$ be the Boolean algebra of four elements. Consider the $L$-fuzzy ideal $J: \mathbf{N} \rightarrow L$ defined as follows:

$$
\begin{aligned}
J(x) & =1 & & \text { if } x \in 6 \mathbf{N}, \\
& =\alpha & & \text { if } x \in 2 \mathbf{N} \sim 6 \mathbf{N}, \\
& =\beta & & \text { if } x \in 3 \mathbf{N} \sim 6 \mathbf{N}, \\
& =0 & & \text { everywhere else. }
\end{aligned}
$$

Clearly, the $L$-fuzzy $h$-ideal $J$ is not prime, though $P(a b)=$ $P(a) \vee P(b)$ holds for all $a, b \in \mathbf{N}$. 
Remark 13. While fuzzifying the condition (I) of "primeness" stated in $\$ 2.1$ three types of products of fuzzy left ideals $A$ and $B$ of $S$, are used in the literature: namely, $A o B, A o_{k} B$, and $A o_{h} B[1-3,5,7]$. They are defined as follows:

$$
\begin{gathered}
A o B(x)=\operatorname{Sup}\{\operatorname{Min}\{A(a), B(b)\} \mid x=a b, a, b \in S\}, \\
\begin{aligned}
A o_{k} B(x)=\operatorname{Sup}\left\{\operatorname{Min}\left\{A(a), B(b), A\left(a^{\prime}\right), B\left(b^{\prime}\right)\right\} \mid x+a b\right. \\
\left.=a^{\prime} b^{\prime} ; a, b, a^{\prime}, b^{\prime} \in S\right\}, \\
A o_{h} B(x)=\operatorname{Sup}\left\{\operatorname{Min}\left\{A(a), B(b), A\left(a^{\prime}\right), B\left(b^{\prime}\right)\right\} \mid x+a b+z\right. \\
\left.=a^{\prime} b^{\prime}+z ; a, b, a^{\prime}, b^{\prime}, z \in S\right\}, \quad \forall x \in S .
\end{aligned}
\end{gathered}
$$

This was needed, because the problem of fuzzification of left ideals, left $k$-ideals, and left $h$-ideals were treated as three separate problems. Theorem 3 allows us to rope all the three in one and leads us to a compact characterization of primeness given in Proposition 11.

A semiprime fuzzy ideal, now defines itself.

Definition 14. An $L$-fuzzy left $h$-ideal $J$ of $S$ is called semiprime, if $J$ is nonconstant and, for all $a \in S$ and $\alpha \in L$, the following condition is satisfied:

$$
J(a s a) \geq \alpha, \quad \forall s \in S \Longrightarrow J(a) \geq \alpha .
$$

It follows that a nonconstant $L$-fuzzy ideal (resp., $k$-ideal, $h$ ideal) $I$ of $S$ is semiprime if and only if $\wedge\{I(a s a) \mid s \in S\}=$ $I(a)$ for all $s \in S$. In case $S$ is commutative hemiring with unity, the above equation is further simplified to $I\left(a^{2}\right)=$ $I(a)$. Analogues of Proposition 9 and Corollary 10 can easily be proved.

Theorem 15. Every nonconstant fuzzy ideal (resp., $k$-ideal, $h$-ideal) of a commutative ring with unity is contained in a minimal prime fuzzy ideal (resp., $k$-ideal, $h$-ideal).

Proof. As usual we prove the result for fuzzy $h$-ideals. Let $J$ be a nonconstant fuzzy $h$-ideal of a commutative ring $S$ with unity and $\mathbf{J}=\{x \in S \mid J(x)>J(1)\}$. Let $\mathbf{P}$ be a prime $h$-ideal containing J. Define a fuzzy ideal $P: S \rightarrow[0,1]$ by

$$
\begin{aligned}
P(x) & =1 \quad \text { if } x \in \mathbf{P} \\
& =J(1) \quad \text { if } x \notin \mathbf{P} .
\end{aligned}
$$

Clearly, $P$ is a prime fuzzy $h$-ideal containing $J$ and, thus, the class $C$ of all prime fuzzy $h$-ideals containing $J$ is nonempty. We partially order $C$ by reverse containment, that is, we define $P \leq P^{\prime}$ if and only if $P^{\prime} \subseteq P$ for all $P, P^{\prime} \in C$, and consider a totally ordered subset $\left\{P^{\lambda} \mid \lambda \in \Lambda\right\}$ of $C$. Then, the set $\left\{P_{\alpha}^{\lambda} \mid \lambda \in \Lambda\right\}$ of the $\alpha$-level cuts of $P^{\lambda}$ is a totally ordered set consisting of prime $h$-ideals (and possibly of $S$ ) for each $\alpha \in[0,1]$. Therefore, $\cap\left\{P_{\alpha}^{\lambda} \mid \lambda \in \Lambda\right\}$ is either a prime $h$-ideal of $S$ or $S$ itself. By Proposition 9, $\cap\left\{P^{\lambda} \mid \lambda \in \Lambda\right\}$ is a prime fuzzy $h$-ideal containing $J$. Since $\cap\left\{P^{\lambda} \mid \lambda \in \Lambda\right\}$ is an upper bound of the family $\left\{P^{\lambda} \mid \lambda \in L\right\}, C$ has a maximal element which, clearly, is a minimal prime fuzzy $h$-ideal containing $J$.
Remark 16. Example 12 will testify that Theorem 15 is not valid when $L \neq[0,1]$, in general.

\section{Prime Radicals of a Fuzzy Ideal}

In this section, we assume $S$ to be a commutative hemiring with unity.

Definition 17. If $J$ is an $L$-fuzzy ideal of $S$, then the intersection of all prime $L$-fuzzy ideals (resp., $k$-ideals, $h$ ideals) of $S$ containing $J$ is called the prime (resp., $k$-prime, $h$-prime) radical of $J$. We denote it by $r(J)$ (resp., $\left.r_{k}(J), r_{h}(J)\right)$. If the set of prime $L$-fuzzy ideals (resp., $k$-ideals, $h$-ideals) of $S$ containing $J$ is empty, we define $r(J)$ (resp., $\left.r_{k}(J), r_{h}(J)\right)$ to be $\chi_{S}$.

Note that $r(J)$, (resp., $\left.r_{k}(J), r_{h}(J)\right)$ is a semiprime fuzzy ideal (resp., $k$-ideal, $h$-ideal) containing $J$. Clearly $r(J) \subseteq$ $r_{h}(J) \subseteq r_{k}(J)$. However, the following examples show that strict containment holds.

Example 18. Let $p$ be a prime integer. Consider $\alpha, \beta \in[0,1]$ and $\beta<\alpha$. Define a fuzzy set $P: \mathbf{N} \rightarrow[0,1]$ by

$$
\begin{aligned}
P(x)=1 & \text { if } x=0, \\
=\alpha & \text { if } x \in p \mathbf{N} \sim\{0\}, \\
=\beta & \text { if } x \notin p \mathbf{N} .
\end{aligned}
$$

By Proposition 9, $P$ is a prime fuzzy ideal (also $k$-ideal and $h$-ideal), for all $0 \leq \beta<\alpha \leq 1$. We will call the fuzzy ideal $P$ a prime fuzzy $k$-ideal induced by the prime number $p$ and denote it by $(p \mathbf{N})^{\alpha \beta}$.

Example 19. Suppose $\alpha, \beta, \gamma \in[0,1]$ and $\gamma<\beta<\alpha$. Define a fuzzy set $Q: \mathbf{N} \rightarrow[0,1]$ by

$$
\begin{aligned}
Q(x)=1 & \text { if } x=0, \\
=\alpha & \text { if } x \in p \mathbf{N} \sim\{0\}, \\
=\beta & \text { if } x \in \mathbf{N} \sim(p \mathbf{N} \cup\{1\}), \\
=\gamma & \text { if } x=1 .
\end{aligned}
$$

By Proposition 9, $Q$ is a prime fuzzy ideal which is neither a fuzzy $k$-ideal nor a fuzzy $h$-ideal, for all $0 \leq \gamma<\beta<\alpha \leq 1$. We will call the fuzzy ideal $Q$ a prime fuzzy ideal induced by the prime integer $p$ and denote it by $(p \mathbf{N})^{\alpha \beta \gamma}$. Note that, in the light of Theorem 7 , these are the only prime fuzzy ideals of $\mathbf{N}$ which are not fuzzy $k$-ideals.

Example 20. Consider a fuzzy ideal defined by $J: \mathbf{N} \rightarrow[0,1]$ :

$$
\begin{aligned}
J(x) & =1 \quad \text { if } x=0, \\
& =0.5 \quad \text { if } x \geq 3, \\
& =0 \quad \text { if } x=1 \text { or } 2 .
\end{aligned}
$$


Let $0 \leq \alpha<1$ and $O^{\alpha}$ be the fuzzy $k$-ideal defined by $O^{\alpha}$ : $\mathbf{N} \rightarrow[0,1]:$

$$
\begin{aligned}
O^{\alpha}(x)=1 & \text { if } x=0 \\
=\alpha & \text { if } x \neq 0
\end{aligned}
$$

Let $X=\left\{O^{\alpha} \mid 0.5 \leq \alpha<1\right\} \cup\left\{(p \mathbf{N})^{\alpha \beta} \mid p\right.$ is prime, $0.5 \leq \beta<\alpha \leq 1\}$ and $Y=\left\{(p \mathrm{~N})^{\alpha \beta \gamma} \mid 0 \leq \gamma \leq 0.5<\right.$ $\beta \leq \alpha \leq 1, p$ is prime . Clearly, $X$ is the set of all prime fuzzy $k$-ideals of $\mathbf{N}$ containing $J$ and $Y$ is the set of all those prime fuzzy ideals containing $J$, which are not fuzzy $k$-ideals. Since $r_{h}(J)=r_{k}(J)=\cap\{P \mid P \in X\}$ and $r(J)=\cap\{P \mid P \in X \cup Y\}$, it is mundane to verify that $r_{h}(J)=r_{k}(J)=O^{0.5}$ and $r(J)$ is the fuzzy ideal defined by $r(J): \mathbf{N} \rightarrow[0,1]$ :

$$
\begin{aligned}
& r(J)(x)=1 \quad \text { if } x=0, \\
& r(J)(x)=0.5 \quad \text { for } x \geq 2, \\
& r(J)(x)=0 \quad \text { if } x=1 .
\end{aligned}
$$

Clearly, $r(J) \subset r_{k}(J)=r_{h}(J)$.

Example 21. Let $S=\{0, \alpha, \beta, 1\}$ be the Boolean algebra of four elements. Consider the prime fuzzy ideal defined by $J$ : $S \rightarrow[0,1] J(0)=1=J(\alpha)$ and $J(\beta)=J(1)=0.5$. Then, $J$ being a prime fuzzy $k$-ideal $r_{k}(J)=J$. Since all the fuzzy ideals of $S$ are fuzzy $k$-ideals, we have $r(J)=r_{k}(J)$. Since the set of fuzzy $h$-ideals of $S$ is empty, $r_{h}(J)=\chi_{S}$.

Clearly, $r(J)=r_{k}(J) \subset r_{h}(J)$.

\section{Nil Radicals of a Fuzzy Ideal}

In this section, we assume $S$ to be a commutative hemiring with unity.

Recall that if $I$ is an ideal of $S$, then its radical (also called nil radical) is defined as $\sqrt{ } I=\left\{x \in S \mid x^{n} \in I\right.$, for some integer $n>0$.

We define the fuzzy analogue of nil radical as follows.

Definition 22. If $J$ is an $L$-fuzzy ideal of $S$, then the $L$-fuzzy set $\sqrt{ } J: S \rightarrow L$ defined by $\sqrt{ } J(x)=\vee\left\{J\left(x^{n}\right) \mid n>0\right\}$ is called the $L$-fuzzy (nil) radical of $J$.

Through series of propositions we prove that, when $L$ is totally ordered and $J$ is a fuzzy $k$-ideal (resp., $h$-ideal) of $S$, so is $\sqrt{ } J$.

The following results are the direct consequences of Definition 22.

Proposition 23. If $I$ is an ideal of $S$, then $\sqrt{ }\left(\chi_{I}\right)=\chi \sqrt{ } I$, where $\chi_{I}$ and $\chi \sqrt{ } I$ are the characteristic functions of $I$ and $\sqrt{ } I$, respectively.

Proposition 24. If $P$ is a prime L-fuzzy ideal, then $\sqrt{ } P=P$.

Proposition 25. If $J$ and $K$ are L-fuzzy ideals of a hemiring, then, the following statements hold.
(a) $\sqrt{ }(\sqrt{ } J)=\sqrt{ } J$.
(b) If $J \subseteq K$, then $\sqrt{ } J \subseteq \sqrt{ } K$.
(c) $\sqrt{ }(J \cap K)=\sqrt{ } J \cap \sqrt{ } K$.

Proposition 26. Let J be an L-fuzzy ideals of $S$ and $0 \leq \alpha<1$. Then, the following statements hold.

(i) $\sqrt{ }\left(J_{\alpha}\right) \subseteq(\sqrt{ } J)_{\alpha}$.

(ii) If $L$ is a totally-ordered set, then $(\sqrt{ } J)_{\alpha+}=\sqrt{ }\left(J_{\alpha+}\right)$, where $J_{\alpha+}$ and $(\sqrt{ } J)_{\alpha+}$ are strict level cuts.

Proof. We prove only (ii):

$$
\begin{aligned}
x \in(\sqrt{ } J)_{\alpha+} & \Leftrightarrow \sqrt{ } J(x)>\alpha \\
& \Leftrightarrow \vee\left\{J\left(x^{n}\right) \mid n>0\right\}>\alpha \\
& \Leftrightarrow J\left(x^{n}\right)>\alpha \quad \text { for some } n>0 \\
& \Leftrightarrow x^{n} \in J_{\alpha+} \text { for some } n>0 \\
& \Leftrightarrow x \in \sqrt{ }\left(J_{\alpha+}\right) .
\end{aligned}
$$

The following example shows that the set inclusion in Proposition 26(i) can be strict.

Example 27. Let $\mathbf{N}$ be the hemiring of non-negative integers, $L=[0,1]$, and $p \in \mathbf{N}$ a prime number. Let $\left\langle p^{n}\right\rangle$ denote the ideal of $\mathbf{N}$ generated by $p^{n}$.

Define a fuzzy ideal as follows:

$$
\begin{gathered}
J: \mathbf{N} \longrightarrow L \\
J(x)=0, \quad \text { if } x \notin\langle p\rangle, \\
J(x)=\frac{n}{n+1}, \quad \text { if } x \in\left\langle p^{n}\right\rangle \sim\left\langle p^{n+1}\right\rangle, n=1,2, \ldots \\
J(0)=1 .
\end{gathered}
$$

Since $\sqrt{ } J(p)=\sup \{n /(n+1) \mid n=1, \ldots\}=1$, we have $p \in$ $(\sqrt{ } J)_{1}$. On the other hand, for each $n, J\left(p^{n}\right)=(n /(n+1))<1$ and thus, $p^{n} \notin J_{1}$, for any $n$. Consequently, we have $p \notin \sqrt{ } J_{1}$.

Theorem 28. If $L$ is totally ordered and $J$ an L-fuzzy $k$-ideal (resp., h-ideal) of $S$, then $\sqrt{ } J$ coincides with $r_{k}(J)\left(\right.$ resp., $\left.r_{h}(J)\right)$.

Proof. As usual we restrict our discussion to $h$-ideals. If $P$ is an $L$-fuzzy prime $h$-ideal containing $J$, then, by Proposition 26, we have $(\sqrt{ } J)_{\alpha+}=\sqrt{ }\left(J_{\alpha+}\right)=r_{h}\left(J_{\alpha+}\right)$, where $r_{h}\left(J_{\alpha+}\right)$ is the $h$-prime radical of the (scrisp) ideal $J_{\alpha+}$. On the other hand, for any $x \in S$ and $n>0$, we have $J\left(x^{n}\right) \leq P\left(x^{n}\right)=$ $P(x)$ and therefore, we have $\sqrt{ } J \subseteq r_{h}(J)$.

Suppose $\sqrt{ } J \neq r_{h}(J)$. Then, there exists $a \in S$, such that $(\sqrt{ } J)(a)<r_{h}(J)(a)$. Let $(\sqrt{ } J)(a)=\alpha$. Since $a \notin r_{h}\left(J_{\alpha+}\right)$, there exists a prime $h$-ideal say $\mathbf{P}$ such that $J_{\alpha+} \subseteq \mathbf{P}$ and $a \notin \mathbf{P}$.

Consider the following prime $L$-fuzzy $h$-ideal:

$$
\begin{gathered}
P: S \longrightarrow L \\
P(x)=1, \quad \text { if } x \in \mathbf{P}, \\
=\alpha, \quad \text { if } x \notin \mathbf{P} .
\end{gathered}
$$


Clearly, if $x \in \mathbf{P}$, then we have $J(x) \leq P(x)$. On the other hand, if $x \notin \mathbf{P}$, then we have $x \notin J_{\alpha+}$ and $J(x) \leq \alpha=P(x)$. Thus, we get $J \subseteq P$ and consequently, $r_{h}(J) \subseteq P$.

However, this leads to the following contradiction, $\sqrt{ } J(a)<r_{h}(J)(a) \leq P(a)=\alpha=\sqrt{ } J(a)$.

Hence, we have $\sqrt{ } J=r_{h}(J)$.

Corollary 29. Let $L$ be a totally ordered set. If $J$ is an $L-f u z z y$ $k$-ideal (resp., $h$-ideal) of $S$, then, so is $\sqrt{ } J$.

\section{Correspondence Theorems}

In this section, $f: S \rightarrow S^{\prime}$ is a homomorphism of hemirings, $J$ is an $L$-fuzzy left ideal of $S$, and $J^{\prime}$ is an $L$-fuzzy left ideal of $S^{\prime}$.

In [20, Proposition 3.11], Zhan claims that if $J$ is an $L$ fuzzy $h$-ideal with sup property, then $f(J)$ is an $L$-fuzzy $h$ ideal of $f(S)$. The following example does not substantiate the claim.

Example 30. Let $S$ be the hemiring given in Example 1 (b), $\mathbf{N}$ be the hemiring of non-negative integers, and $f: \mathbf{N} \rightarrow S$ be the epimorphism given by $f(x)=\min \{x, 3\}$ for all $x \in \mathbf{N}$.

Define a mapping $J: \mathbf{N} \rightarrow[0,1]$ by

$$
\begin{aligned}
J(x) & =1 \quad \text { if } x=0, \\
& =\frac{1}{2} \quad \text { if } x=2 n \neq 0, \\
& =0 \quad \text { if } x=2 n+1, \quad \forall n \in \mathbf{N} .
\end{aligned}
$$

Since $f(x)=x$ for all $x \leq 3$ and $f(x)=3$ for all $x \geq 3$, it can be verified that $f(J)(0)=1, f(J)(2)=f(J)(3)=1 / 2$, and $f(J)(1)=0$.

One can readily see that $J$ is an $L$-fuzzy $h$-ideal with sup property; but $f(J)$ is not an $L$-fuzzy $h$-ideal. For, we have $1 \oplus$ $2 \oplus 3=0 \oplus 3$ and $f(J)(2) \wedge f(J)(0) \not \leq f(J)(1)$.

Example 30 raises a natural question: What are the sufficient conditions for a homomorphic image of an $h$-ideal (resp., $k$-ideal) to be an $h$-ideal (resp., $k$-ideal)? In order to answer this question, we introduce the following definition.

Definition 31. Let $f: S \rightarrow S^{\prime}$ be a homomorphism of hemirings. An $L$-fuzzy left ideal $J$ of $S$ is called $f$-compatible if, for all $x, a, b, z \in S, f(x+a+z)=f(b+z) \Rightarrow J(x) \geq$ $J(a) \wedge J(b)$.

Recall that $J$ is $f$-invariant, if $f(a)=f(b)$ implies $J(a)=$ $J(b)$. We leave it to the reader to prove that an $f$-compatible fuzzy left ideal is $f$-invariant.

Proposition 32. Let $f: S \rightarrow S^{\prime}$ be a homomorphism of hemirings and $J$ and $J^{\prime}$ L-fuzzy left ideals of $S$ and $S^{\prime}$, respectively. Then, the following statements hold.

(1) $f^{-1}\left(J^{\prime}\right)$ is an $f$-invariant L-fuzzy left ideal of $S$.

(2) If $J^{\prime}$ is an L-fuzzy left $k$-ideal, then so is $f^{-1}\left(J^{\prime}\right)$.

(3) If $J^{\prime}$ is an L-fuzzy left h-ideal, then $f^{-1}\left(J^{\prime}\right)$ is an $f$ compatible L-fuzzy left $h$-ideal of $S$.
(4) If $J$ is $f$-invariant (in particular if $J$ is f-compatible), then $f(J) f(x)=J(x)$ and therefore, $f^{-1}(f(J))=J$.

(5) If $f$ is an epimorphism, $f\left(f^{-1}\left(J^{\prime}\right)\right)=J^{\prime}$.

Proof. We prove (4) and (5). If $J$ is $f$-invariant and $x \in$ $S$, then it is obvious that $f(J) f(x)=\vee\{J(z) \mid f(z)=$ $f(x)\}=J(x)$. This proves (4). Moreover, if $x^{\prime}=f(x)$, then $f\left(f^{-1}\left(J^{\prime}\right)\right)\left(x^{\prime}\right)=f^{-1}\left(J^{\prime}\right)(x)=J^{\prime}(f(x))=J^{\prime}\left(x^{\prime}\right)$. It, then, follows that if $f$ is an epimorphism, then $f\left(f^{-1}\left(J^{\prime}\right)\right)=$ $J^{\prime}$.

This leads to the following correspondence theorem for $L$-fuzzy left $k$-ideals and $h$-ideals.

Theorem 33. Let $f: S \rightarrow S^{\prime}$ be an epimorphism of hemirings.

(1) There is one-to-one correspondence between the set of $L$-fuzzy left ideals (resp., $k$-ideals) of $S^{\prime}$ and that of $f$ invariant L-fuzzy left ideals (resp., $k$-ideals) of S.

(2) There is one-to-one correspondence between the set of $L$-fuzzy left $h$-ideals of $S^{\prime}$ and that of $f$-compatible $L$ fuzzy left h-ideals of $S$.

The above correspondence preserves prime and semiprime $L$ fuzzy left ideals (resp., $k$-ideals, $h$-ideals).

Proof. Suppose Jand $J^{\prime}$ are $L$-fuzzy left ideals of $S$ and $S^{\prime}$. By Proposition 32, the correspondence is given by $J \leftrightarrow f(J)$ and $J^{\prime} \leftrightarrow f^{-1}\left(J^{\prime}\right)$. We only need to verify that, when $J$ is an $L$ fuzzy left ideal (resp., $k$-ideals, $h$-ideal), then so is $f(J)$, under the conditions specified for $J$. A reader may easily prove that, when $J$ is $f$-invariant, $f(J)$ is an $L$-fuzzy left ideal. Let, moreover, $J$ be an $L$-fuzzy $k$-ideal, $x^{\prime}+a^{\prime}=b^{\prime}, f(x)=x^{\prime}$, $f(a)=a^{\prime}$, and $f(b)=b^{\prime}$. Then $f(x+a)=f(b)$ and therefore, we have $J(x+a)=J(b)$. Let $\alpha=J(a) \wedge J(b)$ and consider $J_{\alpha}$. Clearly, $a, b \in J_{\alpha}$. Since $J(x+a)=J(b)$, we have $x+a \in J_{\alpha}$ and $J_{\alpha}$ being a $k$-ideal $x \in J_{\alpha}$. Therefore, $J(x) \geq J(a) \wedge J(b)$. But, by Proposition $32(4)$, this inequality is equivalent to $f(J)\left(x^{\prime}\right) \geq f(J)\left(a^{\prime}\right) \wedge f(J)\left(b^{\prime}\right)$. Thus, $f(J)$ is an $L$-fuzzy left $k$-ideal.

On similar lines, one can prove that, when $J$ is an $f$ compatible $L$-fuzzy left $h$-ideal of $S, f(J)$ is an $L$-fuzzy left $h$-ideal of $S^{\prime}$.

\section{Primary Fuzzy Ideals}

In this section, we assume that $S$ is a commutative hemiring with unity.

Recall that an ideal $\mathbf{Q}$ of a hemiring $S$ is primary, if (i) $\mathbf{Q} \neq S$ and (ii) $x y \in \mathbf{Q} \Rightarrow x \in \mathbf{Q}$ or $y^{n} \in \mathbf{Q}$ for some positive integer $n$.

We define primary fuzzy ideal as follows.

Definition 34. A nonconstant $L$-fuzzy ideal $/ k$-ideal $/ h$-ideal $Q$ of $S$ is primary, if $Q(x y)=Q(x)$ or $Q(x y) \leq Q\left(y^{n}\right)$ for some positive integer $n$.

The following propositions are immediate consequences of Definition 34. 
Proposition 35. A nonconstant L-fuzzy ideal of $S$ is a primary $L$-fuzzy ideal (resp., $k$-ideal, $h$-ideal) if and only if each of its nonempty level cuts is either a primary ideal (resp., $k$-ideal, $h$ ideal) of $S$ or $S$ itself.

Proposition 36. Let $\mathbf{Q}$ be an ideal of $S$. The characteristic function $\chi_{\mathbf{Q}}$ is a primary L-fuzzy ideal (resp., $k$-ideal, $h$-ideal) of $S$ if and only if $\mathbf{Q}$ is primary ideal (resp., $k$-ideal, $h$-ideal), for every complete Heyting algebra $L$.

Proposition 37. Every prime L- fuzzy ideal (resp., $k$-ideal, $h$ ideal) is a primary L-fuzzy ideal (resp., $k$-ideal, $h$-ideal).

The fuzzy ideal $J$ in Example 27 is primary but not prime, as every nonempty, proper level-cut of the fuzzy ideal is primary but not prime.

The proof of the following proposition is straightforward.

Proposition 38. Let $f: S \rightarrow S^{\prime}$ be a homomorphism of hemirings and $Q$ and $Q^{\prime} L$-fuzzy ideals of $S$ and $S^{\prime}$, respectively.

(a) If $Q^{\prime}$ is primary, then $f^{-1}\left(Q^{\prime}\right)$ is primary f-invariant.

(b) Let $f$ be an epimorphism and $Q$ be f-invariant. If $Q$ is a primary, then $f(Q)$ is primary.

(c) If $f$ is an epimorphism and $Q$ is an $f$-compatible primary fuzzy h-ideal, then $f(Q)$ is a primary L-fuzzy $h$-ideal.

Thus, the correspondence theorems in the previous section preserve primary fuzzy ideals as well.

\section{Minimum Imperative for Fuzzification}

In this paper, we fuzzified the concepts of prime ideal, semiprime ideal, and primary ideal of a hemiring. Some of these concepts have been fuzzified earlier in different ways. Therefore, it is pertinent to ask: What constitutes "proper fuzzification" of a concept? Our answer is the following:

Suppose $I$ is a (crisp) ideal with property $p$ of a hemiring $S$ and $\widetilde{I}$ is its fuzzification which inherits property $\widetilde{p}$. If it is the best fuzzification, it should satisfy the following properties.

(1) For every Heyting algebras $L$, the characteristic function of $I$ satisfies the property $\tilde{p}$ if and only if $I$ has property $p$.

(2) $\tilde{I}$ satisfies the property $\tilde{p}$, whenever every nonempty levelcut of $\tilde{I}$ different from $S$ satisfies the property $p$ and conversely.

(3) The set $\widetilde{I}(S)$ has more than two elements. Let $f: S \rightarrow S^{\prime}$ be a homomorphism of hemirings.

(4) If an $L$-fuzzy ideal $J^{\prime}$ of $S^{\prime}$ has property $\tilde{p}$, then $f^{-1}\left(J^{\prime}\right)$, as an $L$-fuzzy ideal of $S$, has property $\tilde{p}$.

(5) If $L$-fuzzy ideal $J$ of $S$ has property $\tilde{p}$, then $f(J)$ is an $L$-fuzzy ideal of $f(S)$ with property $\tilde{p}$, under some preassigned condition(s). The condition(s) is (are) suggested by the corresponding crisp situation.

The last sentence needs some elaboration. If I is a (crisp) ideal of $S, f(\mathbf{I})$ is an ideal of $f(S)$ provided that it satisfies the condition: $f(x)=f(y)$ and $y \in \mathbf{I}$ implies $x \in \mathbf{I}$. The $f$-invariance of $J$ stated above is a "fuzzification" of the condition on the crisp ideal I. If $\mathbf{I}$ is an $h$-ideal, then $f(\mathbf{I})$ is an $h$-ideal of $f(S)$ provided that it satisfies the condition: $f(x+a+z)=f(b+z)$ and $a, b \in \mathbf{I}$ implies $x \in \mathbf{I}$. The $f$ compatibility is a "fuzzification" of the condition on the ideal I.

As proved earlier, the different types of prime, semiprime, and primary $L$-fuzzy ideals defined in this paper fulfill the above five conditions and, therefore, they are the best fuzzifications of the concepts.

\section{Acknowledgments}

The author expresses his deep sense of gratitude to the Editorial Board in general, and Editor-in-Chief in particular, of the journal "Advances in Fuzzy Systems". This paper may not have come out in the present form, if the author has not been invited by them to write it within a specified time limit. $\mathrm{He}$ is also grateful to the referees for their useful suggestions.

\section{References}

[1] J. Ahsan, K. Saifullah, and M. F. Khan, "Fuzzy semirings," Fuzzy Sets and Systems, vol. 60, no. 3, pp. 309-320, 1993.

[2] T. K. Datta and B. K. Biswas, "Fuzzy k-ideals of semirings," Bulletin of Calcutta Mathematical Society, vol. 87, pp. 91-96, 1995.

[3] P. Dheena and S. Coumaressane, "Fuzzy 2-(0- or 1-)prime ideals in semirings," Bulletin of the Korean Mathematical Society, vol. 43, no. 3, pp. 559-573, 2006.

[4] W. A. Dudek, M. Shabir, and R. Anjum, "Characterizations of hemirings by their h-ideals," Computers and Mathematics with Applications, vol. 59, no. 9, pp. 3167-3179, 2010.

[5] S. Ghosh, "Fuzzy k-ideals of semirings," Fuzzy Sets and Systems, vol. 95, no. 1, pp. 103-108, 1998.

[6] H. V. Kumbhojkar, "Spectrum of prime L-fuzzy h-ideals of a hemiring," Fuzzy Sets and Systems, vol. 161, no. 12, pp. 17401749, 2010.

[7] J. Zhan and W. A. Dudek, "Fuzzy h-ideals of hemirings," Information Sciences, vol. 177, no. 3, pp. 876-886, 2007.

[8] H. V. Kumbhojkar and M. S. Bapat, "Correspondence theorem for fuzzy ideals," Fuzzy Sets and Systems, vol. 41, no. 2, pp. 213219, 1991.

[9] H. V. Kumbhojkar and M. S. Bapat, "On prime and primary fuzzy ideals and their radicals," Fuzzy Sets and Systems, vol. 53, no. 2, pp. 203-216, 1993.

[10] H. V. Kumbhojkar and M. S. Bapat, "On semiprime fuzzy ideals," Fuzzy Sets and Systems, vol. 60, no. 2, pp. 219-223, 1993.

[11] D. S. Malik and J. N. Mordeson, "Fuzzy prime ideals of a ring," Fuzzy Sets and Systems, vol. 37, no. 1, pp. 93-98, 1990.

[12] J. N. Mordeson, "Generating properties of fuzzy algebraic structures," Fuzzy Sets and Systems, vol. 55, no. 1, pp. 107-120, 1993.

[13] J. N. Mordeson and P. S. Nair, Fuzzy Matehmatics, Physica, Warsaw, Poland, 1998.

[14] T. K. Mukherjee and M. K. Sen, "On fuzzy ideals of a ring I," Fuzzy Sets and Systems, vol. 21, no. 1, pp. 99-104, 1987. 
[15] U. M. Swamy and K. L. N. Swamy, "Fuzzy prime ideals of rings," Journal of Mathematical Analysis and Applications, vol. 134, no. 1, pp. 94-103, 1988.

[16] J. Ahsan and K. Saifullah, "Semigroups characterized by their fuzzy ideals," Tech. Rep. 147, King Fahd University of Petroleum and Minerals, Dhahran, Saudi Arabia , 1993.

[17] H.V. Kumbhojkar, "On prime fuzzy ideals of a semigroup," in Proceedings of the 26th Annual Iranian Mathematics Conference, pp. 193-199, 1995.

[18] N. Kuroki, "Fuzzy semiprime ideals in semigroups," Fuzzy Sets and Systems, vol. 8, no. 1, pp. 71-79, 1982.

[19] M. K. Sen and M. R. Adhikari, "On maximal k-ideals of semirings," Proceedings of the American Mathematical Society, vol. 118, pp. 699-703, 1993.

[20] J. Zhan, "On properties of fuzzy left h-ideals in hemirings with t-norms," International Journal of Mathematics and Mathematical Sciences, vol. 2005, no. 19, pp. 3127-3144, 2005. 

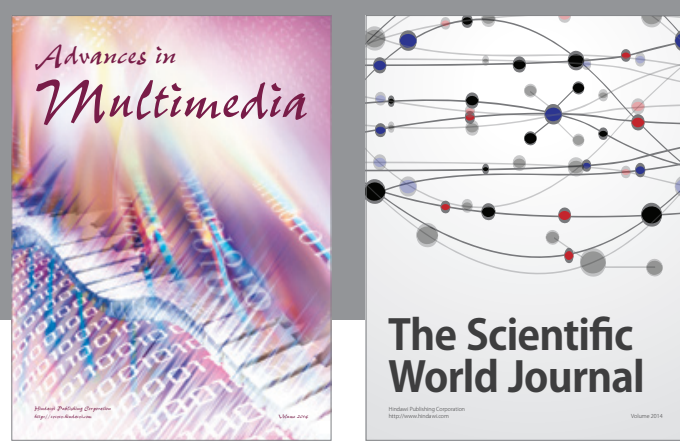

The Scientific World Journal
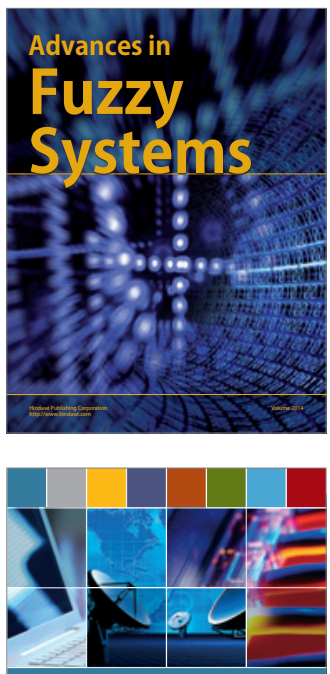

Computer Networks and Communications
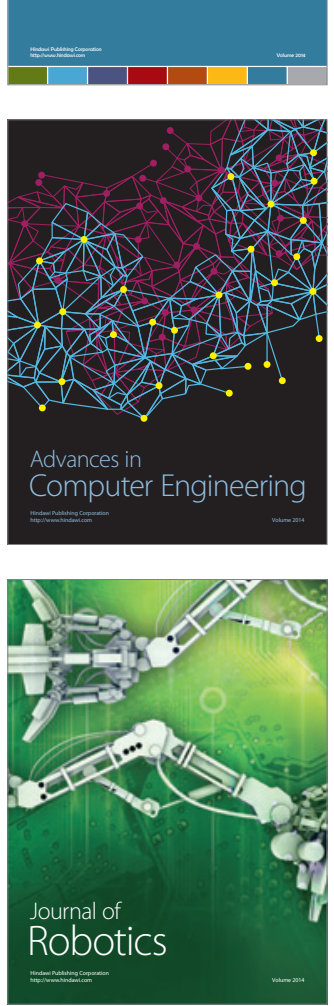
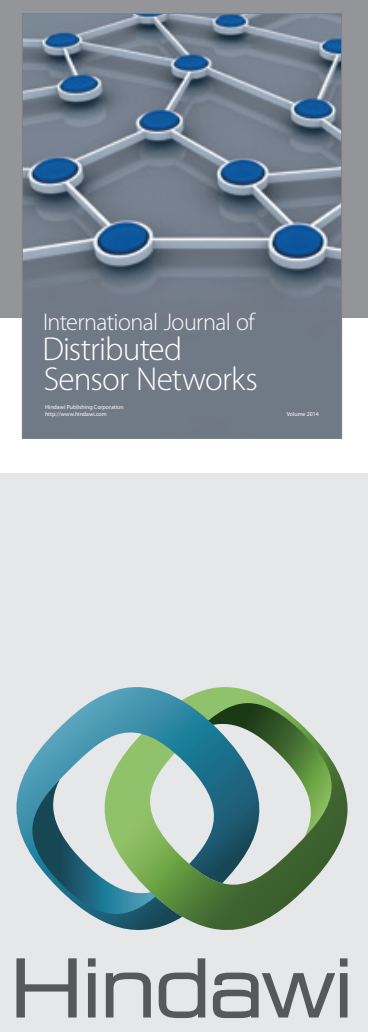

Submit your manuscripts at

http://www.hindawi.com
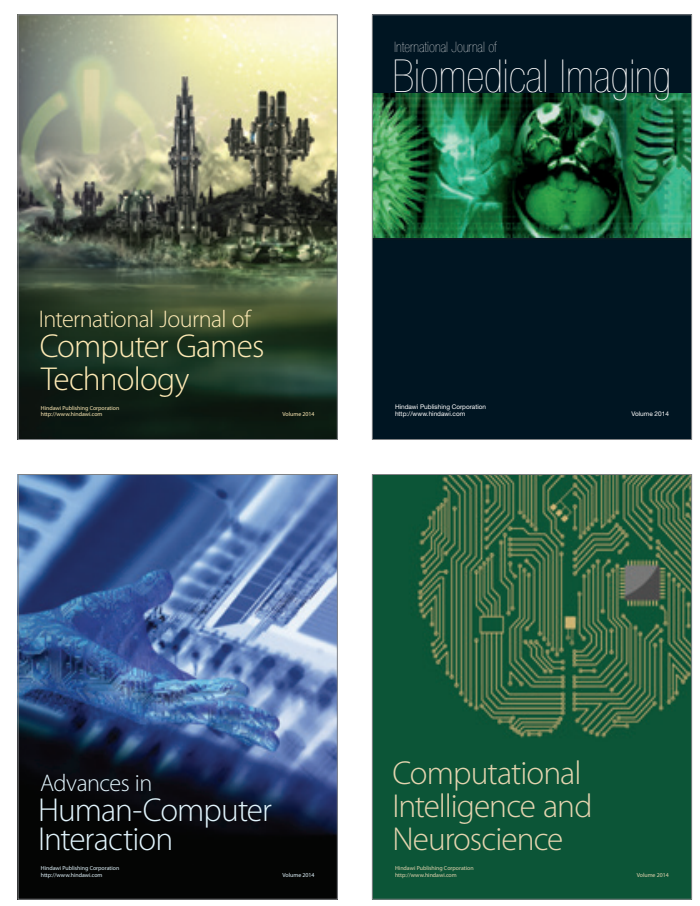
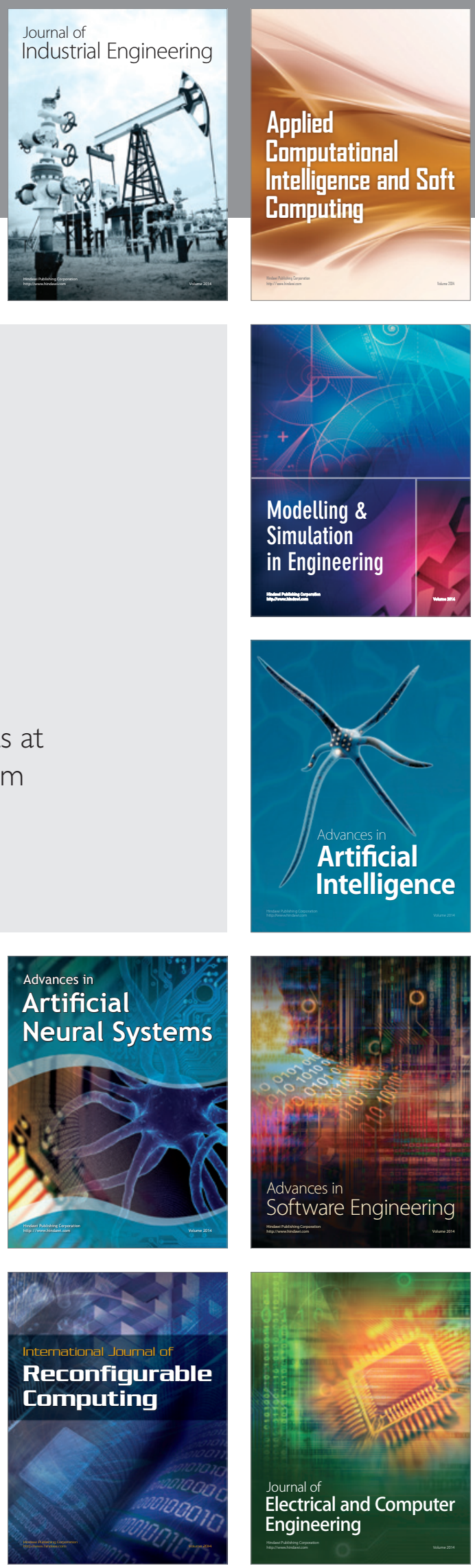